\author{
Military Technical College \\ Kobry El-Kobbah, \\ Cairo, Egypt
}

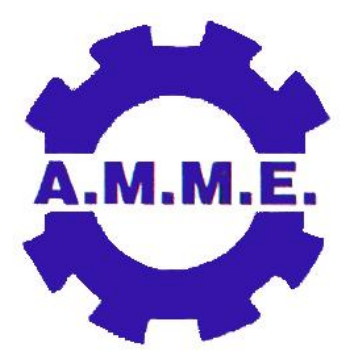

$14^{\text {th }}$ International Conference on Applied Mechanics and Mechanical Engineering.

\title{
Vibration of Fractionally Damped Beams Subjected to a Moving Harmonic Load
}

\author{
By \\ R. Abu-Mallouh ${ }^{*}, 1$ \\ I. Abu-Alshaikh ${ }^{*}$
}

\section{Abstract:}

This paper presents the transverse vibration of Bernoulli-Euler homogeneous isotropic damped beams. These beams are subjected to a harmonic load moving with constant velocity. The damping characteristics of the beams are described in terms of a fractional derivative of arbitrary orders. In the analysis where the initial conditions are assumed to be homogeneous, the Laplace transform cooperates with the decomposition method to find the analytical solution of the handled problems. Subsequently, curves are plotted to show the dynamic response of two beams under different sets of parameters including different orders of the fractional derivatives. The curves reveal that the dynamic response increases as the fractional derivative order becomes greater than unity. This yields that smaller the order of the fractional derivative, the more oscillations the beam suffers. Finally, the literature reviews had shown a good command of agreement with the results obtained in this paper.

\section{Keywords:}

Fractional damping, Moving load, Vibration, Beams, Fractional derivative.

\footnotetext{
${ }^{1}$ Department of Mechanical and Industrial Engineering, Applied Sciences University, Amman 11931 -Jordan
} 


\section{Introduction:}

The general problem of predicting the transverse vibrations of continuous media resulting from the passage of harmonic moving loads is of considerable practical interest in the dynamics of structures. When the subject matter is differential equations of fractional order, it is worth to mention that many physical problems were adequately described by this type of differential equations. The list of these problems is long and the areas of applications are broad. Among these applications are polymeric damping, fluid mechanics, and theory of viscoelasticity [1-5]. However, as noted by these authors and from other literature, a clear physical interpretation of the fractional derivative has been elusive. Good examples are: 1) In the diffusion process, fractional differential equations have been employed to describe an anomalous diffusion regime, including both sub-diffusion and super-diffusion [6]. 2) Many linear viscoelastic damping materials exhibit a macroscopic constitutive behavior, which has been the subject of many investigations involving fractional order derivative [see ref. 7 and references there in]. Transverse vibration of homogeneous beams whose damping behavior is described by a fractional derivative of arbitrary orders was investigated in [8-9]. Agrawal [8] investigated the analytical scheme for stochastic analysis of a continuous beam whose damping characteristics are described by a fractional derivative model of order half. The analytical solution of an initially stationary oscillator subjected to a unit step or impulse excitation were the damping is described by a fractional derivative model of order a half was performed in [9].

This paper presents a homogeneous isotropic fractionally damped beams with the two classical boundary conditions; hinged-hinged and free-fixed, subjected to a harmonic moving load. The basic dynamic characteristics of the beams are obtained based on the assumption of the moving harmonic force model. The solutions are obtained by decomposing the transverse displacement of the beam in the Laplace transform domain. Hence, the basic idea of this method is to decompose the dependent variable which is the generalized displacement into an infinite series. The components of the series are then found recursively and the problem is solved using the inverse Laplace transform of the decomposed equations.

\section{Formulation of the Problem:}

Transverse vibrations of Bernoulli-Euler homogeneous isotropic fractionally damped beams with the two classical boundary conditions; hinged-hinged and free-fixed, subjected to a harmonic load moving with a uniform velocity from left to right are investigated. The beams are assumed to be of finite length and originally at rest. The governing equation of the beam under investigation whose damping characteristics are described by a fractional derivative of order $\beta$ can be written as

$$
E I \frac{\partial^{4} w(x, t)}{\partial x^{4}}+C \frac{\partial^{\beta} w(x, t)}{\partial t^{\beta}}+\mu \frac{\partial^{2} w(x, t)}{\partial t^{2}}=P(x, t),
$$

where $E, I, \mu$ and $C$ are, respectively, the modulus of elasticity, moment of inertia of crosssectional area, mass per unit length, and the coefficient of external damping of the beam. The 
damping is assumed to be light viscous damping. It is also assumed that $E, I, C$ and $\mu$ are constants. Furthermore, $P(x, t)$ is the applied moving harmonic load, and $w(x, t)$ is the transverse deflection of the beam at point $x$ and time $t$. In modal form, the transverse deflection of the beam is written as

$$
w(x, t)=\sum_{n=1}^{\infty} Y_{n}(t) X_{n}(x)
$$

where $Y_{n}(t)$ is the generalized displacement or the modal response of the beam, $X_{n}(x)$ are the normal modes of the un-damped free vibration of the beam written as [10]

$$
X_{n}(x)=\sin \kappa_{n} x+A_{n} \cos \kappa_{n} x+B_{n} \sinh \kappa_{n} x+C_{n} \cosh \kappa_{n} x,
$$

which satisfy the following differential equation

$$
E I \frac{\partial^{4} X_{n}(x)}{\partial x^{4}} Y_{n}(t)+\mu \frac{\partial^{2} Y_{n}(t)}{\partial t^{2}} X_{n}(x)=0,
$$

where the constants $A_{n}, B_{n}$ and $C_{n}$ define the shape and amplitude of the beam vibration. They are evaluated by considering the boundary conditions associated with each beam. The constant $\kappa_{n}$ is the frequency parameter associated with each beam. Substituting equation (2) into equation (1), then multiplying by $X_{m}(x)$ and integrating over the domain (Considering the orthogonality condition), yields the differential equation of the $n^{\text {th }}$ mode of the generalized displacement or the modal response as

$$
\frac{d^{2} Y_{n}(t)}{d t^{2}}+2 \omega_{n} \zeta_{n} \frac{d^{\beta} Y_{n}(t)}{d t^{\beta}}+\omega_{n}^{2} Y_{n}(t)=\frac{Q_{n}(t)}{M_{n}},
$$

Where

$$
\begin{aligned}
& \omega_{n}=\kappa_{n}^{2} \sqrt{E I / \mu} \quad, \quad \zeta_{n}=C / 2 \mu \omega_{n}, \\
& Q_{n}(t)=\int_{0}^{L} X_{n}(x) P(x, t) d x \quad, \quad M_{n}=\int_{0}^{L} \mu X_{n}^{2}(x) d x
\end{aligned}
$$

are, respectively, the undamped natural circular frequency, damping ratio, generalized load and the mass of beam associated with the $\mathrm{n}^{\text {th }}$ mode. In equation (6), the load $P(x, t)$ which moves on the beam from left to right is written as

$$
P(x, t)=P_{0}(\sin \Omega t) \delta[x-f(t)],
$$

where $\Omega$ is the excitation frequency, $P_{0}$ is the intensity of the applied moving load and $f(t)(f(t)=v t)$ is a function describing the motion of the force at time $\mathrm{t}$, in which $v$ is the constant speed of the moving force. Hence, $Q_{n}(t)$ in equation (6) becomes 


$$
Q_{n}(t)=P_{0} \sin (\Omega t) \int_{0}^{L} X_{n}(x) \delta(x-v t) d x=P_{0} \sin (\Omega t) X_{n}(v t) .
$$

Substituting equation (8) into equation (5) yields

$$
\frac{d^{2} Y_{n}(t)}{d t^{2}}+2 \omega_{n} \zeta_{n} \frac{d^{\beta} Y_{n}(t)}{d t^{\beta}}+\omega_{n}^{2} Y_{n}(t)=\frac{P_{0}}{M_{n}} \sin (\Omega t) X_{n}(v t) .
$$

This solution satisfies the homogeneous initial conditions; $Y_{n}(0)=0$ and $\frac{d Y_{n}(0)}{d t}=0$. To find the exact solution of the second order initial value problem in equation (9), the problem can be dealt with as a linear case; accordingly, the solution $Y_{n}(t)$ can be decomposed into an infinite series of the form

$$
Y_{n}(t)=\sum_{k=0}^{\infty} Y_{n}^{k}(t)
$$

where $Y_{n}^{k}(t) ; k=0,1,2, \ldots$ are the components of $Y_{n}(t)$. The components $Y_{n}(t)$ can be determined recursively by substituting equation (10) into equation (9) as

$$
\begin{aligned}
& \frac{d^{2} Y_{n}^{0}(t)}{d t^{2}}+\omega_{n}^{2} Y_{n}^{0}(t)=\frac{P_{0}}{M_{n}} \sin (\Omega t) X_{n}(v t), \\
& \frac{d^{2} Y_{n}{ }^{k+1}(t)}{d t^{2}}+\omega_{n}^{2} Y_{n}^{k+1}(t)=-2 \omega_{n} \zeta_{n} \frac{d^{\beta} Y_{n}^{k}(t)}{d t^{\beta}}, \quad k \geq 0 .
\end{aligned}
$$

Taking the Laplace transforms of equations (10) and (11), assuming homogeneous initial conditions and using the Laplace transform for the Caputo fractional derivatives as given in [11], we get

$$
y_{n}(s)=\frac{P_{0} \Phi(s)}{M_{n}\left(s^{2}+\omega_{n}^{2}\right)}+\frac{P_{0} \Phi(s)}{M_{n}} \sum_{k=1}^{\infty}(-1)^{k}\left(2 \zeta_{n} \omega_{n}\right)^{k} \frac{s^{\beta k}}{\left(s^{2}+\omega_{n}^{2}\right)^{k+1}} .
$$

where $\Phi(s)=\mathcal{L}\left\{\sin (\Omega t) X_{n}(v t)\right\}$. Taking the inverse Laplace transform of equation (12) and using the convolution theorem we, get 


$$
\begin{aligned}
& Y_{n}(t)=\frac{P_{0}}{\eta_{3} M_{n}}\left[\begin{array}{l}
A_{n}\left(\sin \left(t\left(\omega_{1}+\Omega_{n}\right)\right)\left[\eta_{1}-\eta_{2}\right]-\sin \left(t\left(\omega_{1}-\Omega_{n}\right)\right)\left[\eta_{1}-\eta_{2}\right]\right) \\
+C_{n}\left[\begin{array}{l}
\sin \left(\omega_{n} t\right)\left(-4 \eta_{21}+12 \eta_{24}-8 \eta_{28}+4 \eta_{29}-\eta_{35}\right)+ \\
\eta_{36} \sinh \left(\Omega_{n} t\right) \cos \left(\omega_{1} t\right)-\eta_{31} \cosh \left(\Omega_{n} t\right) \sin \left(\omega_{1} t\right)
\end{array}\right] \\
+B_{n}\left[\begin{array}{l}
\eta_{36} \cos \left(\omega_{n} t\right)+\eta_{36} \cosh \left(\Omega_{n} t\right) \cos \left(\omega_{1} t\right) \\
-\eta_{31} \sinh \left(\Omega_{n} t\right) \sin \left(\omega_{1} t\right)
\end{array}\right]+ \\
{\left[\begin{array}{l}
16 \eta_{12} \cos \left(\omega_{n} t\right)+16 \eta_{4} \cos \left(\omega_{n} t\right)-\eta_{36} \cos \left(\omega_{n} t\right)- \\
\eta_{35} \sin \left(\omega_{n} t\right)-\cos \left(t\left(\omega_{1}+\Omega_{n}\right)\right)\left[\eta_{1}-\eta_{2}\right]- \\
\cos \left(t\left(\omega_{1}-\Omega_{n}\right)\right)\left[\eta_{1}-\eta_{2}\right]
\end{array}\right]+}
\end{array}\right]
\end{aligned}
$$

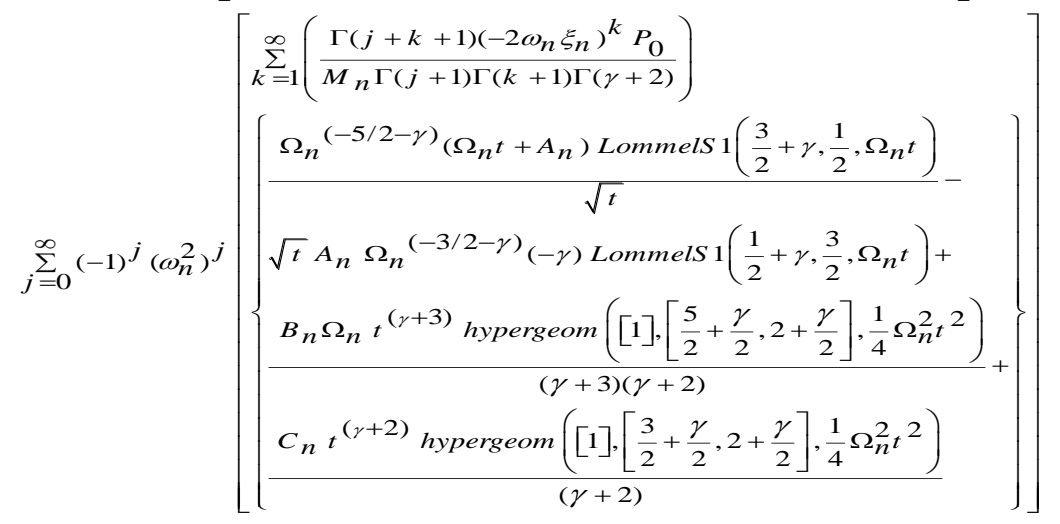

where the values of $\eta_{1}$ to $\eta_{38}$ are given in the appendix In equation (13) LommelS1 (.) and hypergeom (.) are, respectively, the Lommel and generalized hypergeometric functions [12]. Hence, the general transverse deflection of the beam can be found by substituting equations (3) and (13) into equation (2).

In the aforementioned formulation and by introducing the Mittag-Leffler function in two parameters, the method ended with infinite series solution. The second term of this solution, the infinite series term, becomes zero as the generalized damping ratio of the $\mathrm{n}^{\text {th }}$ mode $\zeta_{n}$ goes to zero.

\section{Results and Discussion:}

The analysis outcomes from this paper are applied to homogeneous isotropic fractionally damped beams with the two classical boundary conditions; hinged-hinged and free-fixed. The beams are subjected to concentrated harmonic loads moving with constant velocity. The beams are assumed to be at rest when a harmonic force $P_{0}$ moving according to equation (7) enters the beam from the left-hand side. The instant at which the force arrives at the righthand side of the beam is $t_{f}=L / v$. In Figures (1-2), the dimensionless dynamic deflection

$$
\bar{w}(x, t)=\frac{w\left(x_{\max }, t\right)}{w_{0}},
$$

is shown for all beams versus the dimensionless time parameter $s=\frac{t}{t_{f}}=\frac{t v}{L}$. Thus, when $s=0$, the force is at the left-hand side of the beam, and when $s=1$, the force is at the righthand side of the beam. In the following discussion $w_{0}$ and $x_{\max }$ denote the maximum static deflection and the point on the beam which corresponds to this maximum deflection, respectively. The values for $w_{0}$ and $x_{\max }$ for the hinged-hinged beam are taken as $\frac{P_{0} L^{3}}{48 E I}, \frac{L}{2}$ 
and for the free-fixed beam as $\frac{P_{0} L^{3}}{3 E I}, 0$, respectively. The deflection $w\left(x_{\max }, t\right)$ is obtained from substituting equations (13) and (3) into (2). In these Figures, the effects of damping, speed of the load and the order of the fractional derivative are made clear. The effect of speed is represented by the dimensionless speed parameter $\alpha$ which is defined as

$$
\alpha=v / c_{c r} \quad, \quad c_{c r}=\omega_{1} L / \pi \quad, \quad \Omega=\omega_{1},
$$

where $c_{c r}$ is the critical speed, and $\Omega$ is the excitation frequency defined as [10]. Results presented in Figures (1) and (2) are obtained for three values of $\alpha: \alpha=0.25,0.5$ and 1, three values of the fractional derivative $\beta: \beta=0.25,1$ and 1.75 , and two value of the damping coefficient $\zeta_{n}: \zeta_{n}=0$ and 0.2. Figure 1 (a) and (b) shows the dimensionless dynamic deflection $\left(w\left(x_{\max }, t\right) / w_{0}\right)$ versus the dimensionless time $(s)$ for the two beams under consideration. At this point, it is worth mentioning that the curves of Fig.1 fit exactly those presented in [10,13] for $\beta=1, \zeta_{n}=0$ and $\alpha=0.25, \quad 0.5$ and 1 .
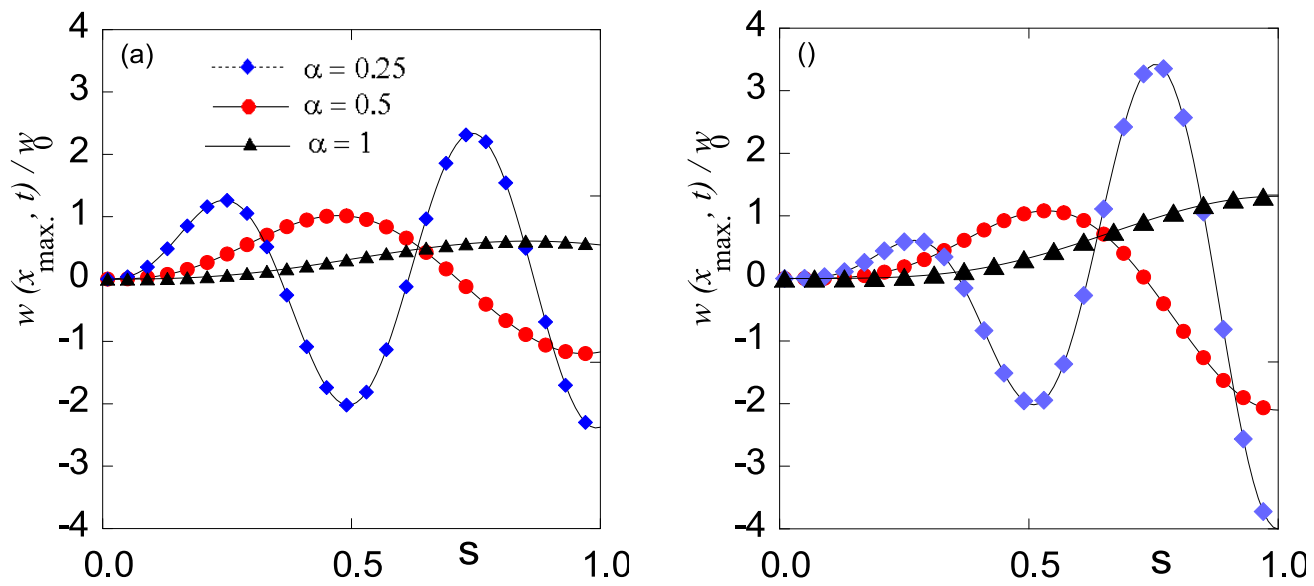

Figure (1): Normalized time variation of dimensionless dynamic deflection $\left(w\left(x_{\max }, t\right) / w_{0}\right)$ for $\alpha=0.25, \alpha=0.5, \alpha=1$ and $\zeta_{n}=0$ for: (a) free-fixed, (b) hinged-hinged beam at the corresponding point of maximum static deflection $x_{\max }$.
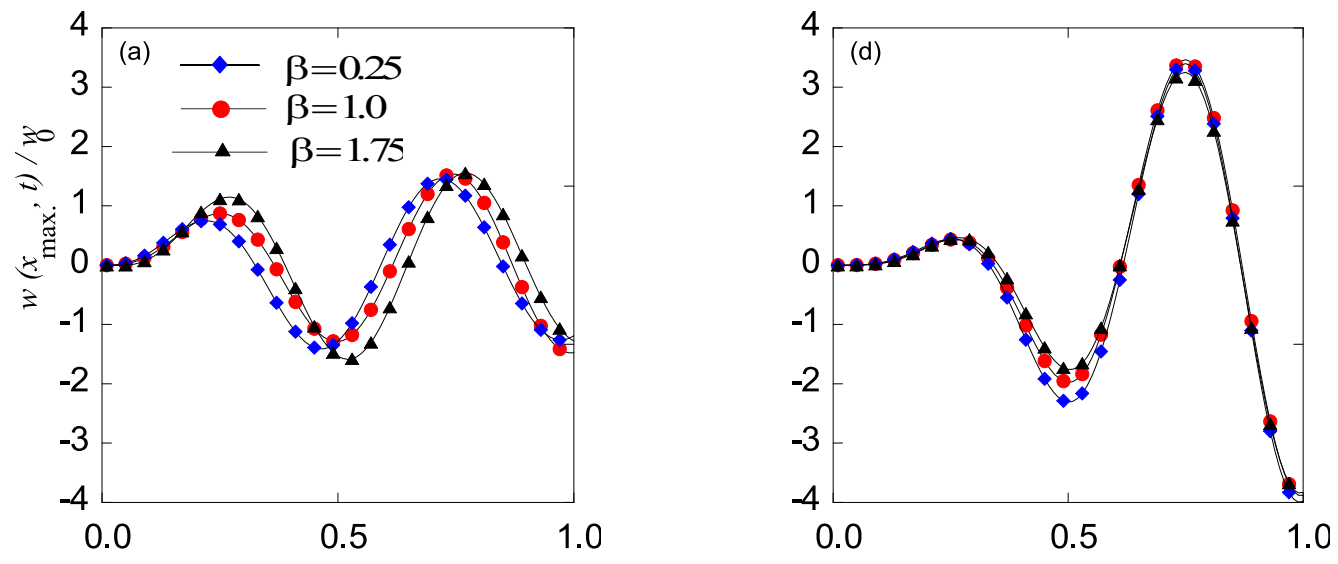

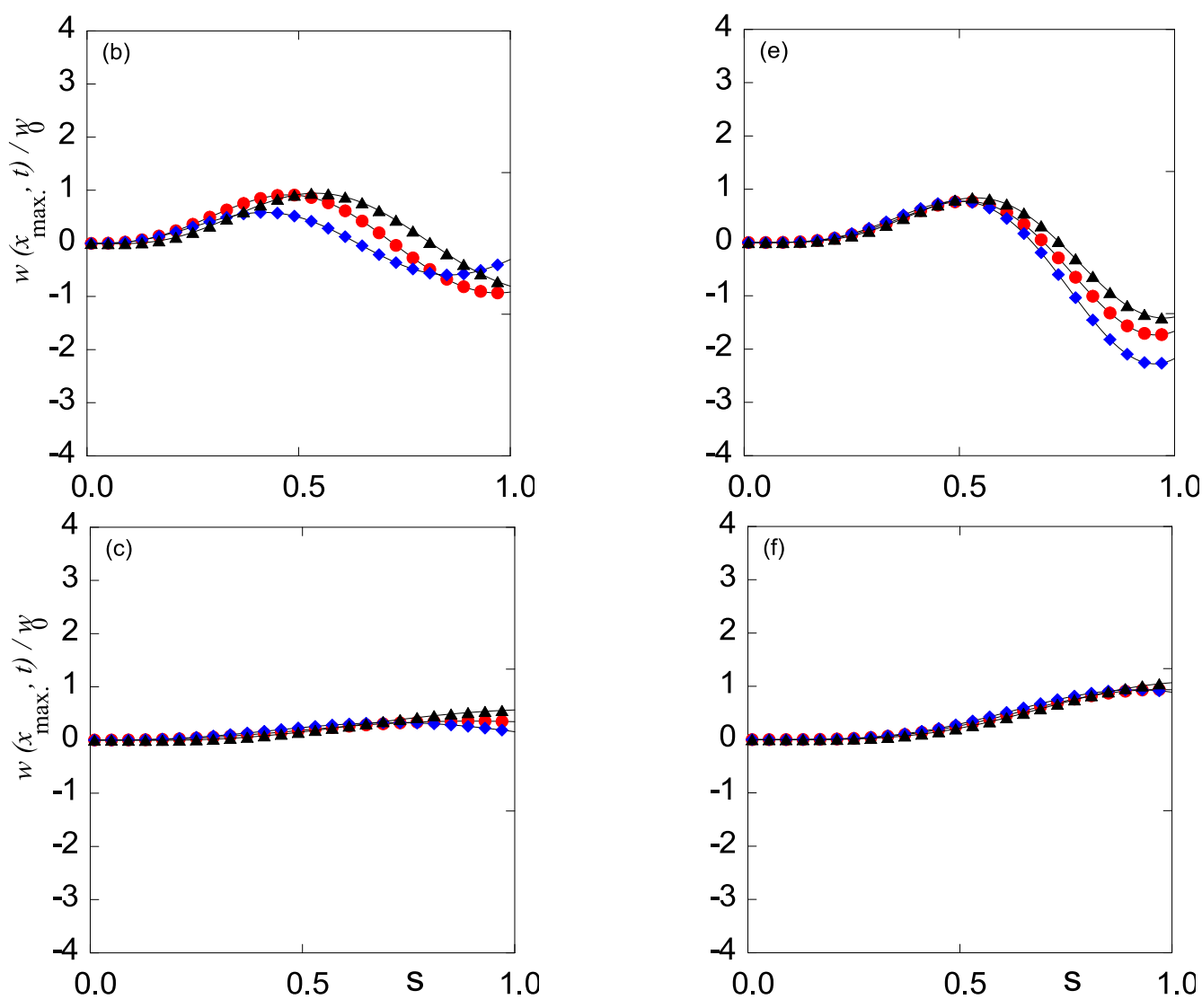

Figure (2): Normalized time variation of dimensionless dynamic deflection $\left(w\left(x_{\max }, t\right) / w_{0}\right)$ for a free-fixed beam at $x_{\max }=0$ for: (a) $\alpha=0.25$, (b) $\alpha=0.5$, (c) $\alpha=1$ and for a hinged hinged beam at $x_{\max }=L / 2$ for: (d) $\alpha=0.25$, (e) $\alpha=0.5$, (f) $\alpha=1$, all values were taken for $\zeta_{n}=0.2$.

Figure 2 shows the dimensionless dynamic deflection $\left(w\left(x_{\max }, t\right) / w_{0}\right)$ versus the dimensionless time $(s)$ for the free-fixed beam (a-c) and for hinged-hinged beam (d-f). Figures $2(\mathrm{a}, \mathrm{d}),(\mathrm{b}, \mathrm{e})$ and $(\mathrm{c}, \mathrm{f})$ are drawn, respectively, for $\alpha=0.25,0.5$ and 1 . In these Figures the effect of the order of the fractional derivative is shown clearly, it is noted that as the order of the fractional derivative increases the amplitude increases and shifts to the right. This means that the smaller the value of the derivative order $\beta$, the more oscillations the beams suffer. A very good agreement is found between the curves of Figure (2) for $\beta=1$ and the corresponding curves presented in [10].

\section{Conclusions:}

An analytical model is presented to solve transverse vibration of Bernoulli-Euler beams with damping characteristics that are described in terms of fractional derivatives of arbitrary order. The method employed is the Laplace transform with the decomposition method to find the analytical solution. Although, the method used has the ability to get the infinite series solution directly by introducing the Mittag-Leffler function. This solution may be written in a closed form for some special cases; otherwise, it may be truncated and fulfilled easily by using mathematical software like Maple. 
Numerical results presented in this paper, show that the dynamic response increases as the damping factor decreases and as the fractional derivative order becomes greater than unity the dynamic response increases. This means that the smaller the value of the fractional derivative order $(\beta)$, the more oscillations the beams suffer. This may allow us to select a suitable mathematical model that precisely prescribes an experimental model; however, this selection may be the fractional derivative model.

\section{References:}

[1] T. Pritz, Five-parameter fractional derivative model for polymeric damping materials, Journal of Sound and Vibration 265(5) (2003), 935-952.

[2] H. Qi and M. Xu, Unsteady flow of viscoelastic fluid with fractional Maxwell model in a channel, Mechanics Research Communications 34(2) (2007), 210-212.

[3] R.L. Bagley and P.J. Torvik, On the fractional calculus model of viscoelastic behavior, Journal of Rheology 30 (1986), 133-135.

[4] N. Shimizu, W. Zhang, Fractional calculus approach to dynamic problems of viscoelastic materials, JSME International Journal, Series C 42 (1999), 825-837.

[5] N. Makris, M.C. Constantinou, Fractional derivative Maxwell model for viscous damper, Journal of Structural Engineering, ASCE 117 (1991), 2708-2724.

[6] Kwok Sau Fa, A falling body problem through the air in view of the fractional derivative approach, Physica A 350 (2005), 199-206.

[7] A. Chatterjee, Statistical origins of fractional derivatives in viscoelasticity, Journal of Sound and Vibration 284(5) (2005), 1239-1245.

[8] Om P. Agrawal, Analytical solution for stochastic response of a fractionally damped beam, Journal of Vibration and Acoustics 126 (2004),561-566.

[9] S. Saha Ray, B. P. Poddar and R. K. Bera, Analytical solution of a dynamic system containing fractional derivative of order one-half by Adomian decomposition method, Journal of Applied Mechanics 72 (2005),290-295.

[10] M. Abu Hilal and M. Mohsen, Vibration of beams with general boundary conditions due to a moving harmonic load, Journal of Sound and Vibration 232 (4) (2000), 703-717.

[11] [33] I. Podlubny, Fractional Differential Equations. Academic Press, 1999.

[12] Y. Luke, The Special Functions and Their Approximations, Volumes 1 and 2. Academic Press, 1969.

[13] L. Fryba, Vibration of Solids and Structures under Moving Loads, Noordhoff International, Groningen, 1972.

\section{Appendix:}




$$
\begin{aligned}
& \gamma=2 j+2 k-k \beta \quad, \quad \eta_{1}=8 \eta_{4}+8 \eta_{12}-\eta_{36} / 2 \quad, \quad \Omega_{n}=\kappa_{n} v \quad, \\
& \eta_{2}=4 \eta_{5}+4 \eta_{14}-8 \eta_{15}+4 \eta_{19}-\eta_{38} / 2-\eta_{37}, \\
& \eta_{3}=\left\{\begin{array}{c}
\omega_{1}^{2}\left[\begin{array}{c}
-48 \Omega_{n}^{4} \omega_{n}^{3}+32 \eta_{19}+32 \eta_{14}-64 \eta_{15}+32 \eta_{5}-48 \eta_{13} \\
-4 C_{3}-16 \eta_{8}+4 \eta_{31}-48 \eta_{17}+48 \eta_{7}-16 \eta_{11}-8 \eta_{36}
\end{array}\right]+ \\
4 \omega_{n}\left(\omega_{n}^{8}-2 \omega_{n}^{4} \Omega_{n}^{4}+\Omega_{n}^{4}+\omega_{1}^{8}-4 \omega_{n}^{2} \omega_{1}^{6}+\left(6 \omega_{n}^{5}-2 \Omega_{n}^{4}\right) \omega_{1}^{4}\right)
\end{array}\right\} \\
& \eta_{4}=\omega_{n}^{3} \Omega_{n}^{3} \omega_{1}, \eta_{5}=\omega_{n}^{5} \Omega_{n} \omega_{1}, \eta_{6}=\omega_{1}^{4} \Omega_{n}^{2} \omega_{n}, \eta_{7}=\omega_{n}^{3} \omega_{1}^{4}, \\
& \eta_{8}=\omega_{n}^{3} \Omega_{n}^{4}, \eta_{9}=\Omega_{n}^{6} \omega_{n}, \eta_{10}=\omega_{n}^{5} \Omega_{n}^{2}, \eta_{11}=\omega_{1}^{6} \omega_{n}, \\
& \eta_{12}=\omega_{1}^{3} \Omega_{n}^{3} \omega_{n}, \eta_{13}=\omega_{1}^{2} \Omega_{n}^{4} \omega_{n}, \eta_{14}=\omega_{1} \Omega_{n}^{5} \omega_{n}, \eta_{15}=\omega_{1}^{3} \Omega_{n} \omega_{n}^{3}, \\
& \eta_{16}=\omega_{1}^{2} \Omega_{n}^{2} \omega_{n}^{3}, \eta_{17}=\omega_{1}^{2} \omega_{n}^{5}, \eta_{18}=\omega_{n}^{7}, \eta_{19}=\omega_{1}^{5} \Omega_{n} \omega_{n}, \eta_{20}=\omega_{1}^{7}, \\
& \eta_{21}=\omega_{1}^{5} \Omega_{n}^{2}, \eta_{22}=\omega_{1}^{3} \Omega_{n}^{4}, \eta_{23}=\omega_{1}^{3} \omega_{n}^{4}, \eta_{24}=\omega_{1} \Omega_{n}^{2} \omega_{n}^{4}, \eta_{25}=\omega_{1} \omega_{n}^{6}, \\
& \eta_{26}=\omega_{1} \Omega_{n}^{4} \omega_{n}^{2}, \eta_{27}=\omega_{1}^{5} \omega_{n}^{2}, \eta_{28}=\omega_{1}^{3} \Omega_{n}^{2} \omega_{n}^{2}, \eta_{29}=\omega_{1} \Omega_{n}^{6}, \eta_{30}=\omega_{1}^{3} \Omega_{n}^{3} \omega_{n}, \\
& \eta_{31}=6 \eta_{13}+2 \eta_{11}+6 \eta_{17}+2 \eta_{8}-6 \eta_{7}+4 \eta_{16}-2 \eta_{9}-6 \eta_{6}+2 \eta_{10}-2 \eta_{18}, \\
& \eta_{32}=8 \eta_{12}+4 \eta_{19}+8 \eta_{4}-8 \eta_{15}+4 \eta_{5}+4 \eta_{14}, \\
& \eta_{33}=\left[\begin{array}{l}
\eta_{8}+3 \eta_{6}-\eta_{18}-2 \eta_{19}+3 \eta_{13}+4 \eta_{15}-3 \eta_{7}-4 \eta_{12}+ \\
3 \eta_{17}-2 \eta_{14}-\eta_{10}+\eta_{11}+\eta_{9}-2 \eta_{5}-4 \eta_{4}-2 \eta_{16}
\end{array}\right], \\
& \eta_{34}=-6 \eta_{7}-\eta_{32} / 2-\eta_{31} / 2+2 \eta_{11}+6 \eta_{17}+6 \eta_{13}-2 \eta_{18}+2 \eta_{8}, \\
& \eta_{35}=-2 \eta_{20}-2 \eta_{21}+2 \eta_{22}-6 \eta_{23}+6 \eta_{24}+2 \eta_{25}+6 \eta_{26}+6 \eta_{27}-4 \eta_{28}+2 \eta_{29} \text {, } \\
& \eta_{36}=16 \eta_{4}-16 \eta_{12}-\eta_{32} \text {, } \\
& \eta_{37}=4 \eta_{16}+\eta_{32} / 2-\eta_{31} / 2-2 \eta_{10}-2 \eta_{9}-6 \eta_{6} \text {, } \\
& \eta_{38}=-16 \eta_{15}+8 \eta_{5}+8 \eta_{19}+8 \eta_{14}-\eta_{32} \text {, }
\end{aligned}
$$

